\title{
Publisher Correction: Complex lasers with controllable coherence
}

Hui Cao@, Ronen Chriki, Stefan Bittner, Asher A. Friesem and Nir Davidson (1)

Nature Reviews Physics (2019) https://doi.org/10.1038/s42254-018-0010-6

Published online 17 January 2019

This article has been corrected to rectify the display of equation 1.

https://doi.org/10.1038/s42254-019-0032-8 I Published online 1 February 2019 